\title{
PSEUDARTROSE DO COLO DO ÚMERO: ANÁLISE DE RESULTADOS DOS CASOS TRATADOS PELA TÉCNICA DESCRITA POR WALCH ET AL
}

\author{
PSEUDARTHROSIS OF THE HUMERAL NECK: ANALYSIS OF \\ THE RESULTS WHEN USING THE TECHNIQUE DESCRIBED BY WALCH ET AL
}

Sérgio Luis Checchia ${ }^{1}$, Alberto Naoki Miyazaki², Marcelo Fregoneze ${ }^{3}$, Pedro Doneux Santos ${ }^{4}$, Luciana Andrade da Silva ${ }^{4}$, Luís Gustavo Prata Nascimento ${ }^{5}$

\section{RESUMO}

Objetivo: Avaliar a consolidação e os resultados funcionais obtidos nos casos de pseudartrose do colo cirúrgico do úmero pelo método de tratamento descrito por Walch et al, em 1996, no qual é utilizado, além do enxerto ósseo esponjoso convencional autólogo e osteossíntese interna com placa e parafusos, um enxerto tricortical intramedular, também autólogo. Métodos: Entre julho de 1997 e maio de 2005 foram tratados, pela técnica descrita, 14 pacientes com o diagnóstico de pseudartrose da extremidade proximal do úmero. Um faleceu no pós-operatório imediato por tromboembolismo pulmonar, sendo reavaliados 13 pacientes. Resultados: Com tempo de seguimento mínimo de 12 e máximo de 130 meses (médio de 51,4 meses), quatro pacientes evoluíram com resultados excelentes, quatro bons e cinco regulares; portanto, houve $61,5 \%$ de resultados excelentes e bons e nenhum ruim. Doze casos (92\%) evoluíram para consolidação, com tempo médio de 3,5 meses. Conclusões: $O$ tratamento cirúrgico da pseudartrose do colo cirúrgico do úmero, por meio da técnica descrita por Walch et al, demonstrou-se eficaz, atingindo $92 \%$ de consolidação; resultados excelentes e bons em $61,5 \%$ dos casos e satisfação dos pacientes, quanto aos resultados finais, em todos os casos; melhor evolução nas pseudartroses decorrentes de fraturas em duas partes do colo cirúrgico quando comparado com as em três partes.

Descritores - Úmero/anatomia; Pseudo-artrose; Fixação interna de fraturas

\section{ABSTRACT}

Objective: To evaluate bone healing and the patient's outcome after open reduction and internal fixation, when using the technique described by Walch et al, in 1996, which uses conventional intramedullary corticocancellous bone graft and internal fixation with plate and screws added by a tricortical intramedullary bone graft, also autologous. Methods: From July 1997 to May 2005, 14 patients were treated by this technique, 14 of these diagnosed with pseudoarthrosis of the humeral proximal end. One died at the early postoperative period due to pulmonary thromboembolism; therefore, 13 patients were re-evaluated. Results: The mean follow-up time was 51.4 months (ranging from 12 to 130 months). Four patients evolved with excellent results, four good and five fair results. Therefore, there were $61.5 \%$ of satisfactory results according to UCLA functional scale and no poor result. Twelve cases (92\%) healed within 3.5 months in average. Conclusion: the surgical treatment of the nonunion of the surgical neck of the humerus using this technique showed an effective outcome with 92\% of healing; excellent and good results in $61.5 \%$ of the cases, as well as satisfaction of all patients with their final results; nonunion resulting from two-part fractures had better results when compared with three-part fractures.

Keywords - Humerus/anatomy; Pseudoarthrosis; Fracture internal, fixation

\footnotetext{
1 - Professor Doutor Adjunto da Faculdade de Ciências Médicas da Santa Casa de São Paulo (FCMSCSP)

2 - Professor Doutor Instrutor da FCMSCSP; Chefe do Grupo de Ombro e Cotovelo do DOT-FCMSCSP

3 - Professor Assistente da FCMSCSP; Instrutor do Grupo de Ombro e Cotovelo do DOT-FCMSCSP.

4 - Médico Assistente do Departamento; Instrutor do Grupo de Ombro e Cotovelo do DOT-FCMSCSP.

5 - Médico, Mestre em Medicina pela FCMSCSP - Grupo de Ombro e Cotovelo do DOT-FCMSCSP.
}

Trabalho realizado no Departamento de Ortopedia e Traumatologia da Faculdade de Ciências Médicas da Santa Casa de São Paulo, Pavilhão "Fernandinho Simonsen". Correspondência: Rua Dr. Cesário Mota Jr., 112 - 01221-020 - São Paulo, SP. Tel./fax: (0xx11) 3222-6866. E-mail: ombro@ombro.med.br 


\section{INTRODUÇÃO}

As fraturas da extremidade proximal do úmero correspondem a aproximadamente $5 \%$ das fraturas do esqueleto humano ${ }^{(1,2)}$. A maioria destas é impactada, sem desvio ou minimamente desviada; geralmente, são tratadas conservadoramente e, na maioria das vezes, obtêmse consolidação da fratura e resultado funcional satisfatório $^{(3-7)}$. Entretanto, com pouca frequência ${ }^{(1,4,5,7-11)}$, podem evoluir para pseudartrose, porém, sua incidência não é bem definida ${ }^{(7,8,12)}$.

Dentre as fraturas da extremidade proximal do úmero, a que mais frequentemente evolui para pseudartrose é a classificada por Neer como em duas partes do colo cirúrgico do úmero ${ }^{(3,4,10,11,13-15)}$; são geralmente incapacitantes devido à dor e perda de movimento ativo da articulação do ombro ${ }^{(1-3,8,10,11,16,17)}$.

O tratamento dessa complicação é dificultado por fatores locais, tais como: osteopenia e reabsorção óssea, tanto pela idade avançada como pelo desuso do membro acometido, além da cavitação no fragmento proximal, como também a comunicação da fratura com o líquido sinovial da articulação e a retração miotendínea e capsuloligamentar ${ }^{(1,5,8,11,16)}$.

Em função dessas dificuldades, diversos tipos de tratamento têm sido propostos para as pseudartroses do colo cirúrgico do úmero: desde o conservador, indicado nos pacientes oligossintomáticos e naqueles com problemas clínicos graves $^{(1,3,5,13,17,18)}$, até os diversos métodos cirúrgicos. Dentre esses podemos citar: a fixação com hastes intramedulares, inicialmente não bloqueadas e sem banda de tensão e, posteriormente, associada a esta, a fixação com haste bloqueada; a osteossíntese interna (OI) com placa e parafusos, associada ou não ao uso de enxerto ósseo autólogo; e as hemiartroplastias que, funcionalmente, propiciam resultados insatisfatórios ${ }^{(13,17,19-22)}$.

Walch et $\mathrm{al}^{(8)}$, descreveram uma técnica na qual associam OI com placa em " $T$ " e enxerto ósseo tricortical intramedular autólogo, além de enxerto esponjoso autólogo periférico ao foco da pseudartrose, obtendo $96 \%$ de consolidação. Com isso, optamos em nossos casos por utilizar esse mesmo princípio descrito por Walch et $\mathrm{al}^{(8)}$; entretanto, modificamos a OI, realizando-a com a placa angulada $P F S 80^{\circledR}$, baseando-se nos seus resultados para fixação de fraturas em duas partes do colo cirúrgico do úmero, que atingiram 91,3\% de excelentes e bons resultados, de acordo com os critérios definidos pela University of Califórnia at Los Angeles (UCLA), e $97,8 \%$ de consolidação ${ }^{(23)}$.
O objetivo deste trabalho é avaliar a consolidação e os resultados funcionais obtidos nos casos de pseudartrose do colo cirúrgico do úmero tratados pela técnica descrita por Walch et $\mathrm{al}^{(8)}$; a OI foi realizada com a placa PFS $80^{\circledR(23)}$.

\section{MÉTODOS}

Entre julho de 1997 e maio de 2005, o Grupo de Ombro e Cotovelo do Departamento de Ortopedia e Traumatologia da Faculdade de Ciências Médicas da Santa Casa de São Paulo tratou 14 pacientes portadores de pseudartrose do colo cirúrgico do úmero.

Como critérios de inclusão, todos os pacientes com pseudartrose do colo cirúrgico do úmero com tempo de seguimento mínimo de um ano e os com possibilidade de terem sido submetidos a essa técnica, ou seja, os casos de pseudartrose decorrentes de fraturas em duas partes do colo cirúrgico do úmero ou dos com consolidação dos tubérculos com desvio menor que cinco milímetros, foram incluídos no trabalho. Portanto, excluímos os casos classificados como complexos, com perda de fragmentos ou ainda os com grande cavitação do fragmento proximal impossibilitando adequada OI. Foi ainda excluído da casuística um paciente que faleceu no período pós-operatório imediato por tromboembolismo pulmonar. Portanto, 13 pacientes foram reavaliados, dos quais nove eram do sexo masculino e quatro, do feminino, com média de idade de 58,5 anos, variando de 25 a 79 anos; o membro dominante foi acometido em sete casos (Tabela 1).

Os mecanismos de lesões foram: queda ao solo em oito casos, acidente automobilístico em dois, acidente motociclístico, atropelamento e trauma direto em um caso cada.

Com relação ao tipo de fratura, de acordo com os critérios de $\mathrm{Neer}^{(24)}, 10$ casos foram classificados em duas partes acometendo o colo cirúrgico e, os outros três casos, em três partes envolvendo colo cirúrgico e tubérculo maior, sendo dois deles fratura-luxação anterior em três partes. Todas as fraturas eram fechadas. Com relação ao tratamento prévio dessas fraturas: sete haviam sido tratadas conservadoramente; quatro, por meio de cirurgia com fios metálicos, um deles tendo sido operado duas vezes, pois evoluiu com perda da redução no período pós-operatório precoce; e dois, com placa angulada $P F S 80^{\circledR(23)}$. A pseudartrose foi diagnosticada, em média, após 7,4 meses da fratura (variando de três a 24 meses) (Tabela 1). 
Tabela 1 - Dados clínicos dos pacientes, tipos de fratura, tratamento da fratura na fase aguda, tempo do diagnóstico da pseudartrose, tipo da pseudartrose, implantes utilizados na cirurgia, tempo de consolidação, mobilidade pós-operatória, tempo de seguimento, resultados funcionais pós-operatórios (UCLA) e complicações.

\begin{tabular}{|c|c|c|c|c|c|c|c|c|c|c|c|c|c|c|}
\hline Caso & Iniciais & Sexo & Idade & Dom. & Tipo de fratura & $\begin{array}{l}\text { Tratamento } \\
\text { fratura }\end{array}$ & T diag. PSA & Tipo PSA & $\begin{array}{c}\text { Implantes na } \\
\text { Cirurgia }\end{array}$ & T cons. & Mob PO & T Seg. & UCLA & Complicações \\
\hline 1 & DR & $\mathrm{F}$ & 61 & + & $2 p(C C)$ & Fios metálicos & $24 \mathrm{~m}$ & Baixa & PFS & $5 \mathrm{~m}$ & $130 / T_{8} / 40$ & $9 a$ & 29 & Soltura da placa \\
\hline 2 & CZM & $\mathrm{F}$ & 79 & & $2 p(C C)$ & Conservador & $3 m$ & Baixa & PFS & $4 m$ & $120 / T_{5} / 40$ & $9 a$ & 27 & \\
\hline 3 & GML & $\mathrm{F}$ & 54 & + & $2 p(C C)$ & Fios metálicos & $3 m$ & Baixa & PFS & $3 m^{1} 1 / 2$ & $160 / T_{5} / 60$ & $7 a$ & 35 & \\
\hline 4 & JFS & M & 51 & & $3 p(C C+G T)$ & Fios metálicos & $4 \mathrm{~m}$ & Baixa & PFS & $4 m^{1} / 2$ & $160 / T_{6} / 45$ & $6 a$ & 35 & \\
\hline 5 & WA & M & 70 & & $2 p(C C)$ & Conservador & $3 m$ & Baixa & PFS + 1 PFO & $2 m 1 / 2$ & $120 / T_{12} / 40$ & 1a & 32 & \\
\hline 6 & RS & M & 25 & & $3 p(C C+G T)$ & PFS & $15 m$ & Baixa & PFS + 1 PFO & $2 m 1 / 2$ & $120 / T_{g} / 30$ & $3 a$ & 28 & Impacto pela placa \\
\hline 7 & $\mathrm{CHK}$ & M & 57 & + & $2 p(C C)$ & Conservador & $8 m$ & Baixa & $P F S+2 P F O$ & $4 m$ & $110 / L_{1} / 40$ & $1 \mathrm{a}$ & 26 & \\
\hline 8 & $\mathrm{DB}$ & M & 66 & & $2 p(C C)$ & Conservador & $4 \mathrm{~m}$ & Baixa & PFS & $2 m$ & $150 / T_{12} / 20$ & $2 a$ & 32 & \\
\hline 9 & DMG & M & 40 & + & $2 p(C C)$ & Conservador & $6 m$ & Alta & PFS & $2 m^{1 / 2}$ & $150 / T_{10} / 20$ & $2 a$ & 35 & \\
\hline 10 & RDN & M & 69 & + & $3 p(C C+G T)$ & Conservador & $7 \mathrm{~m}$ & Baixa & PFS & $4 m$ & $90 / L_{5} / 45$ & $4 a 7 m$ & 27 & \\
\hline 11 & MC & $F$ & 77 & & $2 p(C C)$ & Fios metálicos & $5 \mathrm{~m}$ & Baixa & $\mathrm{PFS}+2 \mathrm{PFO}$ & $5 \mathrm{~m}$ & $130 / T_{12} / 60$ & $2 \mathrm{a} 10 \mathrm{~m}$ & 27 & $\begin{array}{c}\text { Impacto pela } \\
\text { placa+quebra enxerto }\end{array}$ \\
\hline 12 & AT & M & 65 & + & $2 p(C C)$ & PFS & $11 \mathrm{~m}$ & Baixa & $\mathrm{PFS}+1 \mathrm{PFO}$ & $3 m$ & $150 / T_{12} / 45$ & $1 a$ & 34 & \\
\hline 13 & RAA & M & 47 & + & $2 p(C C)$ & Conservador & $3 m$ & Alta & PFS & $5 \mathrm{~m}$ & $120 / T_{8} / 60$ & $1 \mathrm{a} 3 \mathrm{~m}$ & 27 & $\begin{array}{l}\text { Hematoma PO + } \\
\text { falha cons. = reop }\end{array}$ \\
\hline
\end{tabular}

Fonte: DOT-SCMSP

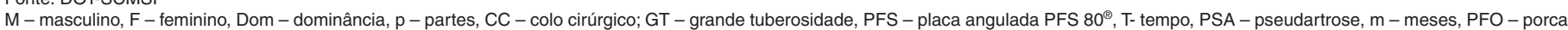
PFO, T cons. - tempo de consolidação, Mob PO - mobilidade pós-operatória, T Seg. - tempo de seguimento, reop. - reoperação

As radiografias utilizadas para o diagnóstico e classificação dessa complicação corresponderam à série de trauma para o ombro: incidência de frente verdadeira em rotação neutra, perfil axilar e perfil de escápula ${ }^{(24)}$. Utili- zamos a classificação de pseudartrose do colo cirúrgico do úmero proposta por Checchia et $\mathrm{al}^{(1)}$ (Figura 1); 11 casos foram classificados como tipo baixa e dois, como do tipo alta (casos 9 e 13) (Tabela 1).
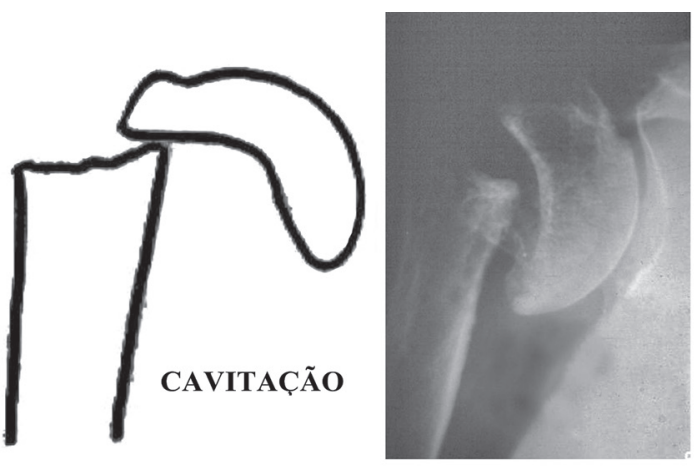

a)

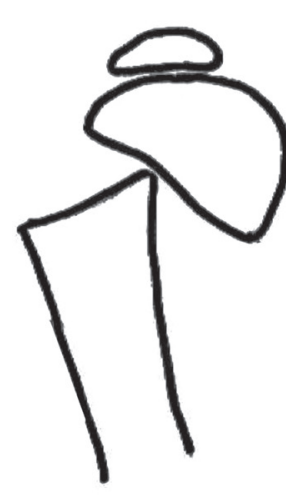

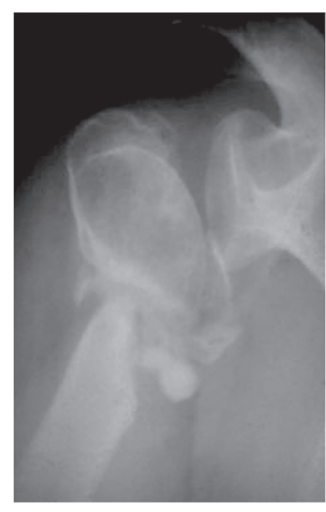

c)
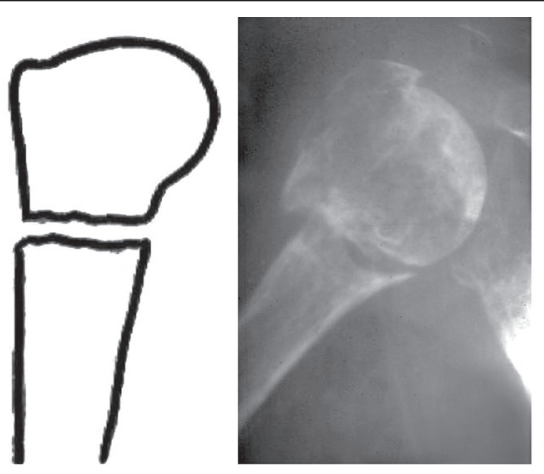

b)
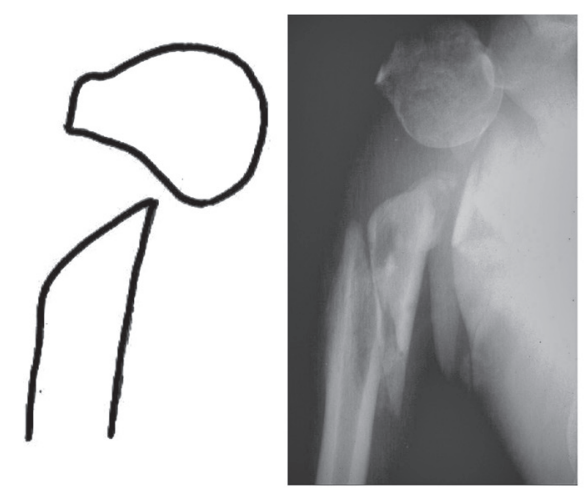

Figura 1 - Classificação de pseudartrose da extremidade proximal do úmero em quatro grupos proposta por Checchia et al: a) Grupo I - em 2 partes TIPO ALTA; b) Grupo II - em 2 partes TIPO BAIXA; c) Grupo III - COMPLEXAS; d) Grupo IV - com PERDA DE FRAGMENTOS. 
A mobilidade no período pré-operatório foi avaliada, porém, seus valores não correspondem à amplitude de movimento da articulação do ombro e, sim, à mobilidade no foco da pseudartrose nesses casos, o que não nos permitiu avaliar o real movimento da articulação.

O método de tratamento cirúrgico utilizado foi a redução aberta e OI com a placa angulada $P F S 80^{\circledR(23)}$, associadas à colocação de enxerto ósseo tricortical intramedular autólogo retirado do ilíaco, além do enxerto ósseo esponjoso autólogo periférico ao foco de pseudartrose, assim como na técnica de Walch et $\mathrm{al}^{(8)}$ (Figura 2). Utilizamos a via de acesso deltopeitoral em todos os casos e, como fonte de enxerto ósseo, a crista ilíaca ântero-superior. O tecido fibroso no foco da pseudartrose foi ressecado e as extremidades ósseas foram cruentizadas para melhorar a circulação sanguínea local. A OI, com a placa angulada $P F S 80^{\circledR(23)}$, foi realizada após o encavilhamento do enxerto tricortical no canal medular diafisário e na cabeça do úmero. Além disso, associamos enxerto ósseo esponjoso autólogo ao redor do foco da pseudartrose em todos os casos (Figura 3). A porca de fixação óssea $\left(P F O^{\circledR}\right)^{*}$ (Figura 4), indicada nos casos de osteopenia local avançada em que a fixação do parafuso junto a cortical é inadequada, foi utilizada em cinco casos (Tabela 1).

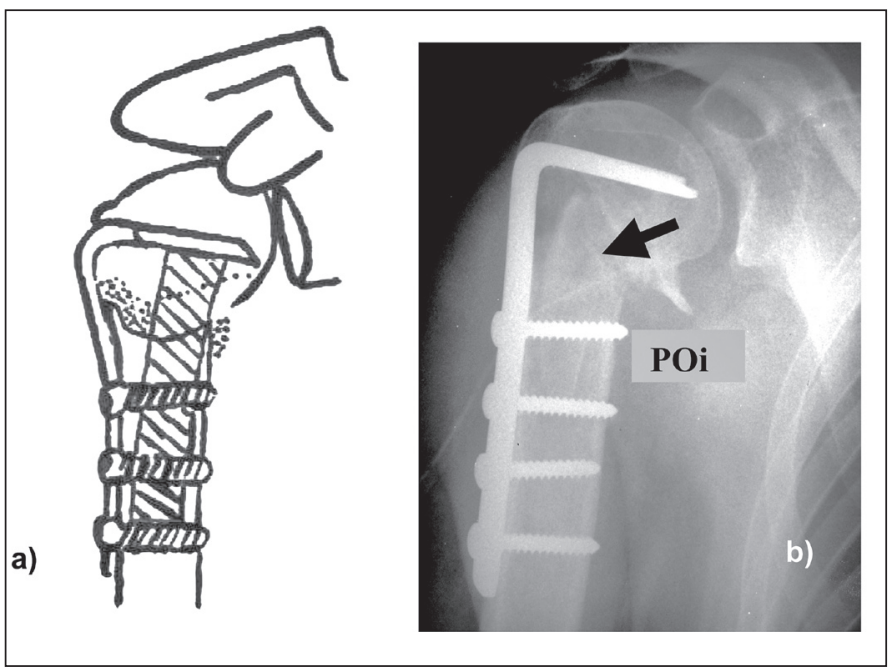

Figura 2 - a) Esquema ilustrativo da técnica de redução aberta e Ol com placa angulada tipo PFS $80^{\circledR}$ associada ao encavilhamento de enxerto tricortical autólogo intramedular retirado da crista ilíaca e enxerto esponjoso periférico ao foco de pseudartrose. b) radiografia do ombro direito, incidência AP, com seta apontando enxerto tricortical intramedular.

No período pós-operatório os pacientes permaneceram com tipoia até ser evidenciada, pelas radiografias, a consolidação da pseudartrose. Até então, eram somente permitidos exercícios ativos com o cotovelo, punho e mão do membro afetado. Após a consolidação, dava-se
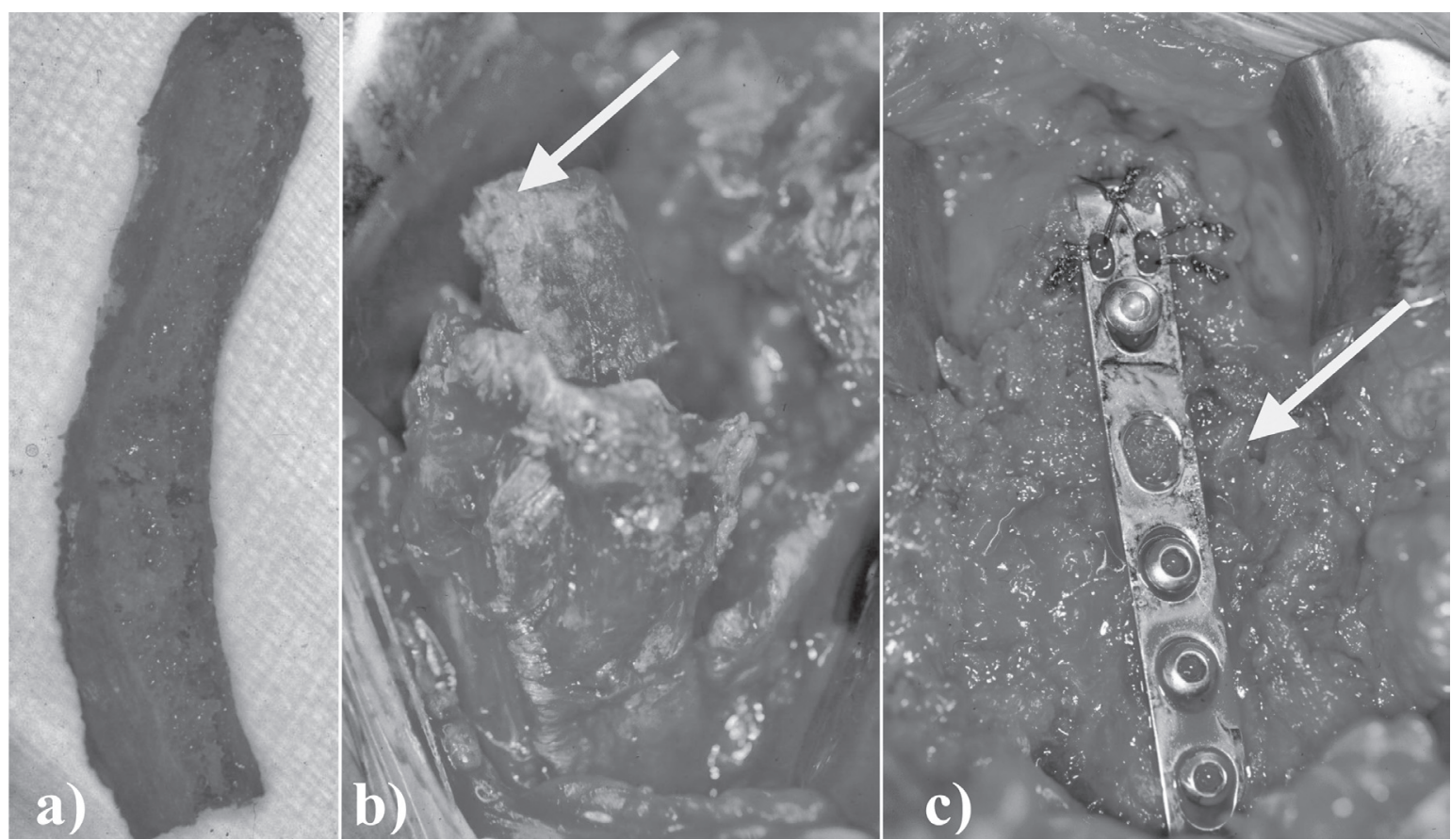

Figura 3 - Ilustração da técnica cirúrgica: a) enxerto retirado da crista ilíaca; b) encavilhamento do enxerto (seta) na diáfise do úmero; c) encavilhamento do enxerto tricortical na cabeça do úmero e fixação com placa PFS $80^{\circ}$ associado ao uso de enxerto esponjoso periférico ao foco da pseudartrose (seta).

\footnotetext{
* Checchia SL, Mercadante MT, Santos PD, Miyazaki NA, Roncetti R, Klausser RG, Waldrigues MG. Desenvolvimento e avaliação preliminar de uma porca para fixação óssea. Tema livre apresentado no $32^{\circ}$ Congresso da Sociedade Brasileira de Ortopedia e Traumatologia realizado de 1 a 4 de novembro de 2000 no Rio de Janeiro.
} 


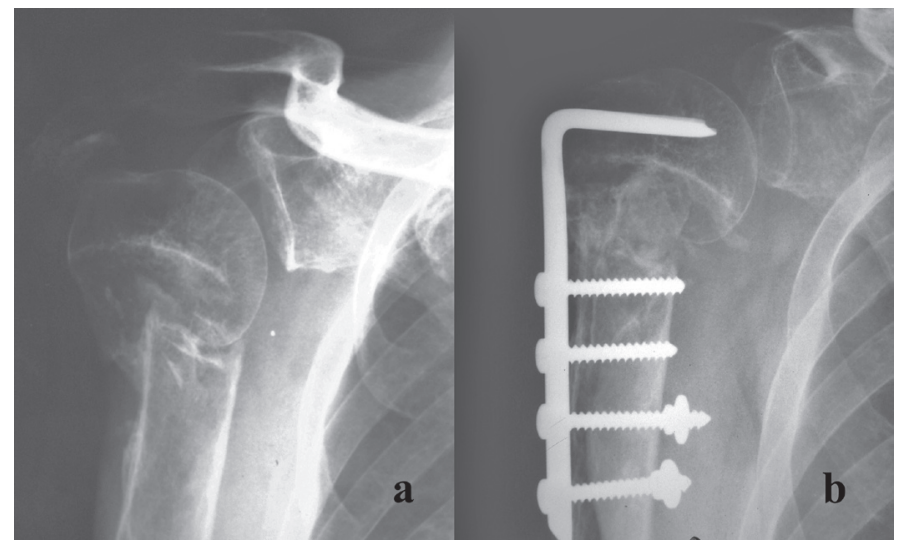

Figura 4 - a) Radiografia na posição ântero-posterior mostrando pseudartrose do colo cirúrgico do úmero; b) radiografia na posição antero-posterior ilustrando a necessidade do uso da porca PFO para melhora na qualidade da fixação no fragmento distal.

início à reabilitação com exercícios passivos e ativos assistidos para ganho de amplitude de movimento, seguidos de exercícios de fortalecimento muscular.

Os pacientes tiveram seus resultados funcionais finais avaliados pelo sistema de pontos definido pela University of Califórnia at Los Angeles (UCLA) ${ }^{(25)}$ e a amplitude de movimento foi medida segundo os critérios descritos por Hawkins e Bokor ${ }^{(26)}$.

Para análise estatística aplicamos o teste de MannWhitney a fim de compararmos os resultados obtidos de acordo com o sexo, idade, o tratamento da fratura na fase aguda, o tipo da fratura inicial e o tipo de pseudartrose, de acordo com a classificação proposta ${ }^{(1)}$. Utilizamos o programa SPSS (Statistical Package for Social Sciences) e adotamos o nível de significância de $5 \%(\mathrm{P}<0,05)$.

\section{RESULTADOS}

Com tempo de seguimento mínimo de 12 e máximo de 130 meses (médio de 51,4 meses), quatro pacientes evoluíram com resultados excelentes, quatro bons e cinco regulares, portanto, verificamos $61,5 \%$ de resultados satisfatórios (Tabela 1). As médias de mobilidade no período pós-operatório foram de $130^{\circ}$ de elevação $\left(90^{\circ}\right.$ a $\left.160^{\circ}\right), 42^{\circ}$ de rotação lateral $\left(20^{\circ}\right.$ a $\left.60^{\circ}\right)$ e T10 de rotação medial (L5 a T5) (Tabela 1).

Comparando os resultados, individualmente, quanto à idade, observamos que os pacientes abaixo de 60 anos de idade obtiveram $66 \%$ de resultados satisfatórios, enquanto os acima de 60 anos, 57\% $(\mathrm{P}=0,735)$, ou seja, sem significância estatística. Quanto ao sexo, $66 \%$ dos homens e $50 \%$ das mulheres evoluíram com resultados satisfatórios, não havendo diferença significante $(\mathrm{P}=0,584)$. Analisando o tratamento prévio da fratura na fase aguda, os pacientes que haviam sido tratados conservadoramente obtiveram $57 \%$ de resultados satisfatórios, enquanto que, dos casos tratados cirurgicamente, $66 \%$ evoluíram satisfatoriamente $(\mathrm{P}=$ 0,735), ou seja, sem significância estatística. Quanto ao tipo de pseudartrose, $54 \%$ das baixas e $100 \%$ das altas tiveram evolução satisfatória, entretanto, sem diferença significante estatisticamente $(\mathrm{P}=0,536)$. Somente com relação ao tipo de fratura houve diferença estatisticamente significativa $(\mathrm{P}=0,025)$, das quais, as em duas partes tiveram $70 \%$ de resultados satisfatórios e, as em três partes, $33 \%$.

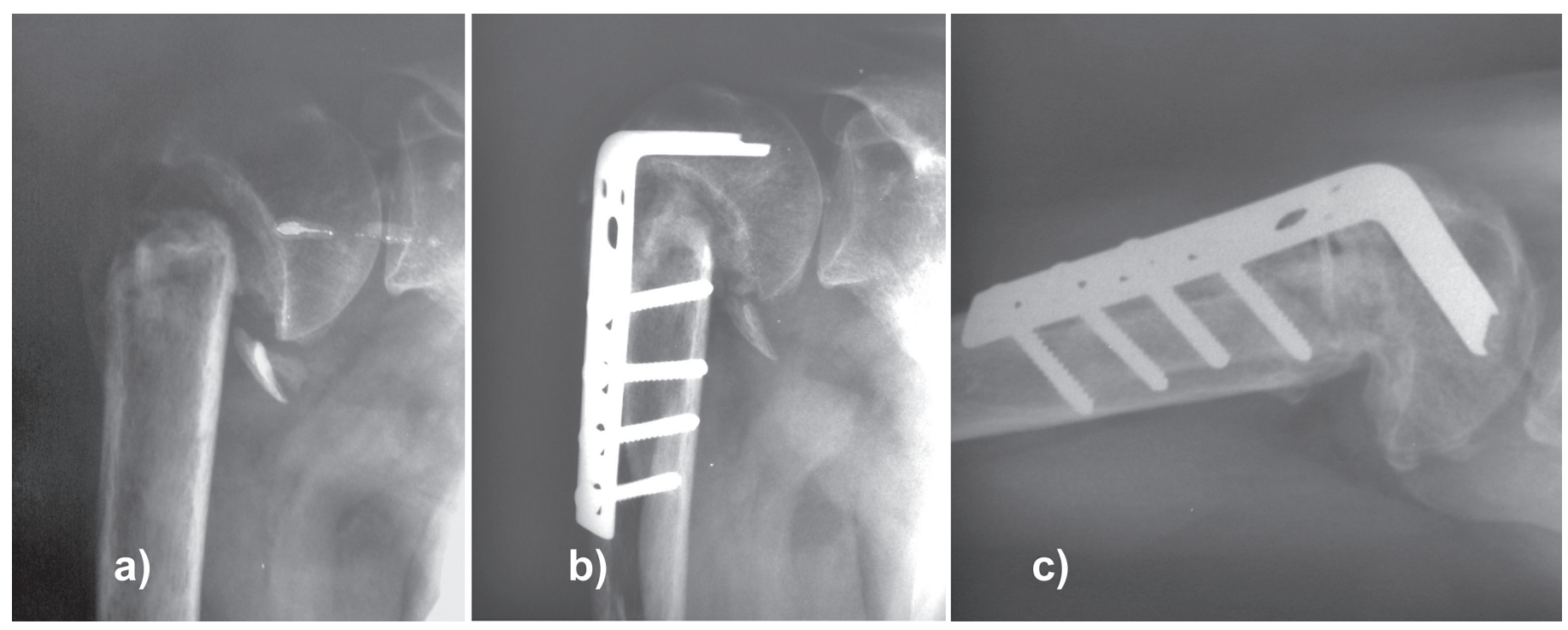

Figura 5 - Caso 13 - radiografia do ombro direito, incidência AP: a) mostrando foco de pseudartrose do colo cirúrgico do úmero; b) imagem no período PO $3 \mathrm{~m}$ do tratamento da pseudartrose mostrando ausência de calo ósseo; c) radiografia do ombro direito, incidência P axilar, no período PO 8m (reoperado) com evidência de consolidação. 
Doze casos evoluíram para consolidação (92\%), com média de tempo de 3,5 meses (dois a cinco meses), e um paciente (caso 13) apresentou, como complicação, suspeita clínica e radiográfica de ausência de consolidação; foi reoperado e observou-se, durante o ato cirúrgico, a consolidação, entretanto, insuficiente, ou seja, ocorrera somente na porção lateral da pseudartrose; foi tratado com adição de enxerto ósseo esponjoso autólogo na falha óssea medial, evoluindo com consolidação completa e UCLA = 27 (Figura 5).

Outras complicações foram observadas em quatro pacientes: um caso de soltura parcial da placa na sua fixação distal, diagnosticada no terceiro mês de pósoperatório, mas que estava consolidada no momento da reavaliação e sem queixa clínica $(\mathrm{UCLA}=29)($ caso 1$)$ (Figuras 6 e 7); impacto subacromial pela placa em dois casos (6 e 11); no primeiro, foi feita a retirada da mesma no período pós-operatório de dois anos e nove meses, evoluindo com bom resultado (UCLA $=28)$. O segundo caso de impacto subacromial havia tido também, como complicação intra-operatória, a quebra do enxerto tricortical, mas que não influenciou o resultado final, consolidando com cinco meses de evolução. Quanto ao impacto pela placa, o paciente optou por não retirá-la, permanecendo com dor, o que fez com que tivesse como resultado final regular (UCLA $=27)$. Por último, tivemos um caso de hematoma (caso 13) diagnosticado na segunda semana de pós-operatório, o qual foi submetido a drenagem (Tabela 1).

\section{DISCUSSÃO}

A pseudartrose do colo cirúrgico do úmero é pouco frequente ${ }^{(1,3-5,7-11)}$, o que é comprovado pela pequena casuística da maioria dos trabalhos publicados na literatura, e, na maioria das vezes, extremamente incapacitante $\mathrm{e}^{(1,3,8,11,12)}$. As fraturas que mais comumente evoluem para pseudartrose são as em duas partes do colo cirúrgi$\mathrm{co}^{(3,4,10,11,13-15)}$, o que foi verificado também pela nossa estatística, em que $76 \%$ acometiam tal região do úmero. Entretanto, devemos observar que, em nosso trabalho, analisamos somente as pseudartroses do colo cirúrgico e as decorrentes de fraturas em três partes com consolidação do tubérculo com desvio menor que cinco milímetros.

Assim como Norris et $\mathrm{al}^{(20)}$, consideramos tempo suficiente para o diagnóstico da pseudartrose o período de três meses após a fratura, pois tivemos quatro casos que, com esse tempo de evolução, já apresentavam sinais de pseudartrose.

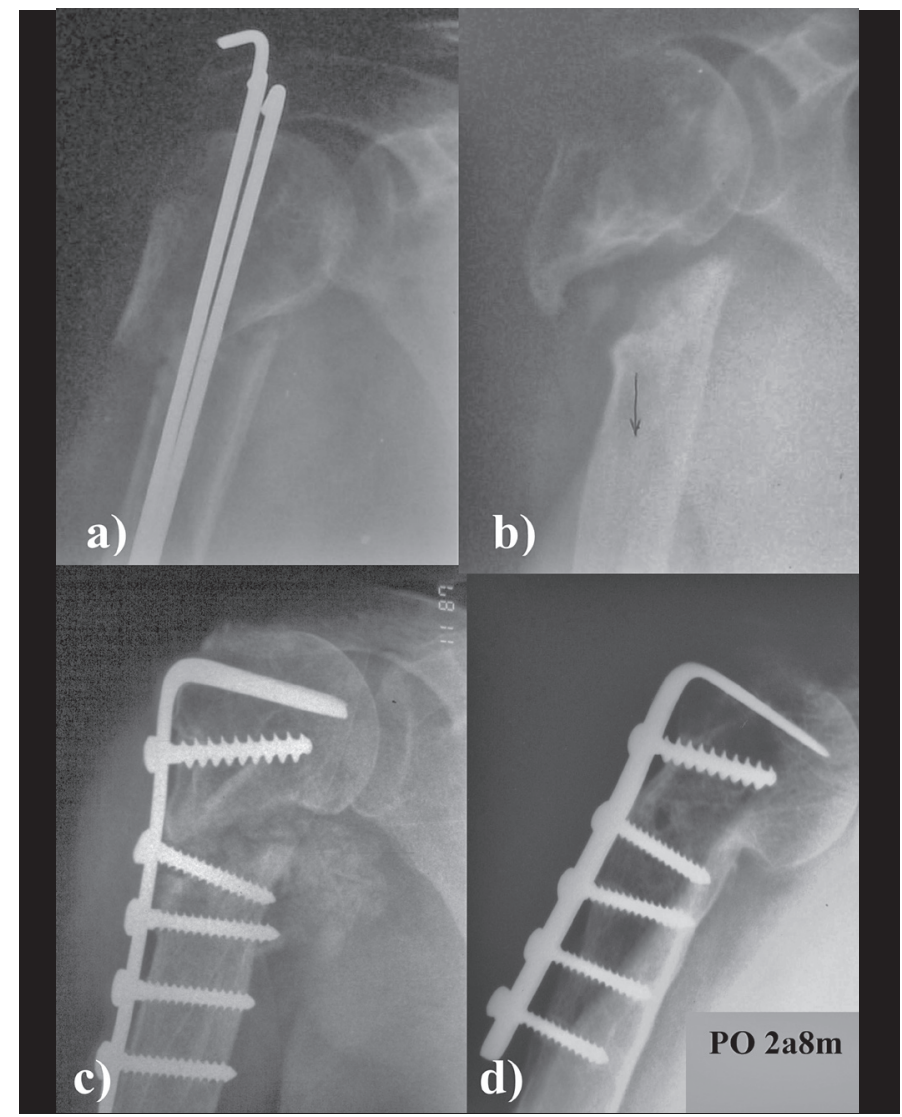

Figura 6 - Caso 1 - radiografias ântero-posteriores: a) fixação da fratura com fios lisos; b) pseudartrose do colo cirúrgico do úmero; c) período pós-operatório imediato de acordo com a técnica descrita por Walch et al; d) período pós-operatório 2 anos e 8 meses mostrando soltura da placa distalmente, mas que evoluiu com consolidação.

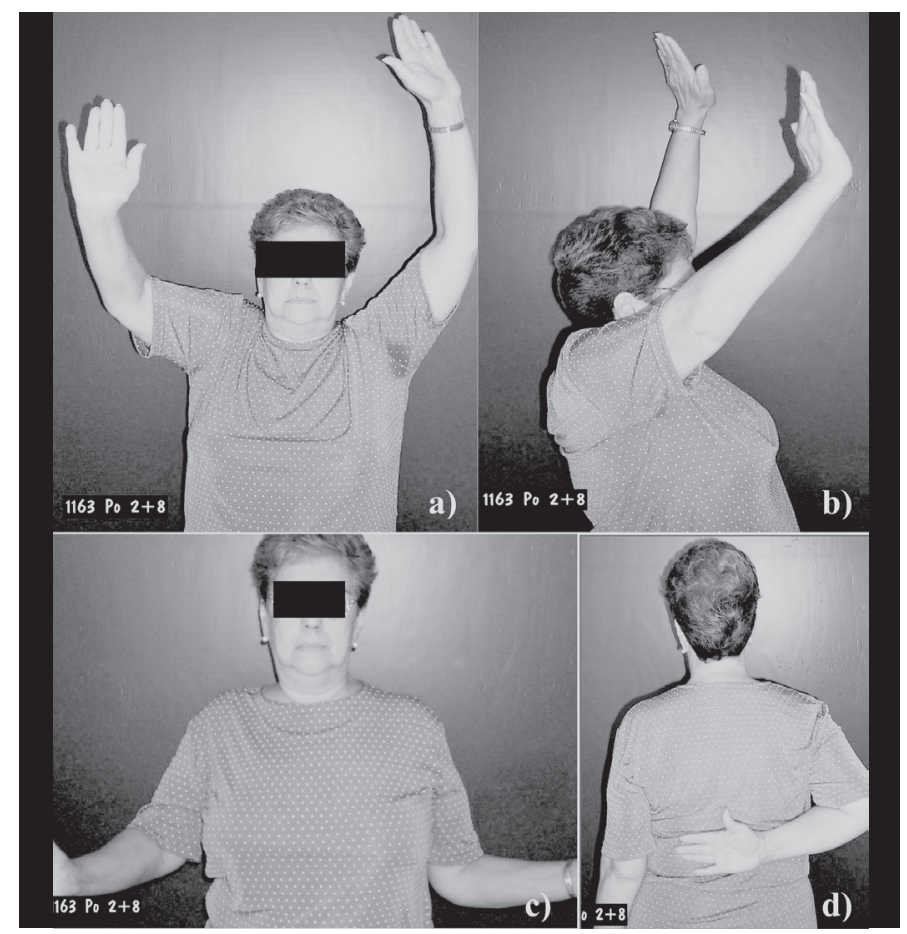

Figura 7 - Caso 1 - fotografias ilustrando aspecto cínico final no período pós-operatório 2 anos e 8 meses: a) elevação vista de frente; b) elevação vista de perfil; c) rotação lateral; d) rotação medial. 
Não analisamos estatisticamente, devido à pequena casuística, o tratamento da fratura na fase aguda como fator relacionado à etiologia da pseudartrose; entretanto, verificamos que os pacientes operados foram, em nossa opinião, tratados de maneira inadequada, predispondo à não consolidação como, por exemplo, os casos 1,3 , 4 e 6 (Tabela 1).

A pseudartrose da extremidade proximal do úmero deve ser tratada o mais precocemente possível para evitar piora das condições locais, como: osteopenia, reabsorção óssea, retração miotendínea e capsuloligamentar e cavitação no fragmento proximal ${ }^{(1,3,5,8,11,16)}$. Entretanto, Duralde et $\mathrm{al}^{(5)}$ não notaram piora dos resultados com o aumento do intervalo entre a fratura e a cirurgia da pseudartrose. A pseudartrose, em nosso estudo, foi diagnosticada, em média, após 7,4 meses da fratura e, apesar de não ser possível comprovar estatisticamente, devido ao pequeno número de casos, não verificamos correlação das pseudartroses com maior tempo de evolução com piores resultados. No entanto, devido às dificuldades locais e alto potencial de complicação, o tratamento conservador também pode ser indicado em idosos, oligossintomáticos e pacientes com problemas clínicos graves ${ }^{(1,3,5,9,13,17,18)}$.

Nos casos em que o fragmento proximal da fratura é muito pequeno, com importante cavitação e porótico, ou seja, nos casos tipo I (alta), está eventualmente indicada a artroplastia parcial ou total, principalmente se houver comprometimento da superfície articular da cavidade glenoidal $^{(1,2,10,21)}$. No entanto, apesar de tal procedimento levar a alívio da dor, ocorre significativa limitação de movimento da articulação do ombro, com prejuízo funcional e resultados finais insatisfatórios ${ }^{(5,13,18,22)}$. De acordo com a classificação utilizada ${ }^{(1)}$, encontramos 11 casos de pseudartroses baixas e apenas duas altas (casos 9 e 13), porém, mesmo nesses casos foi possível realizar a fixação proximal, proporcionando resultado excelente no primeiro $(\mathrm{UCLA}=35)$ e bom no segundo $(\mathrm{UCLA}=28)$ (Tabela 1). Entretanto, em nossa casuística, não havia casos complexos ou em que não fosse possível a OI com a técnica proposta. Nesses casos, em nossa opinião, o que deve ser considerado ao optar pela OI e enxerto ósseo, ao invés da artroplastia, é o fato de, mesmo sendo pseudartrose do tipo alta, haver quantidade óssea mínima que nos permita fixação adequada. Outros autores também consideram que a solução biológica, com síntese estável e enxerto ósseo, é melhor que a artroplastia em situações com adequado estoque ósseo na cabeça do úmero ${ }^{(1,4-6,13,18,20,21)}$.

$\mathrm{Na}$ evolução histórica do tratamento cirúrgico da pseudartrose do colo cirúrgico do úmero, diversas técnicas foram idealizadas para melhora na qualidade da fixação óssea do fragmento proximal. Em 1996, Walch et $\mathrm{l}^{(8)}$ descrevem técnica utilizando fixação interna com placa em " $T$ " associada a enxerto corticoesponjoso intramedular retirado da crista do ilíaco e enxerto esponjoso ao redor, obtendo $96 \%$ de consolidação e $81,2 \%$ na escala de Constant modificada. $\mathrm{O}$ fato de utilizar tal enxerto aumentou a estabilidade da fixação, tanto por atuar como um tutor interno, como pelo aumento da resistência de fixação dos parafusos nos fragmentos proximal e distal. Diferentemente de Walch et $\mathrm{al}^{(8)}$, optamos pela fixação da pseudartrose com placa angulada do tipo $P F S 80^{\circledR(23)}$ baseando-se em seus resultados para fixação de fraturas em duas partes do colo cirúrgico do úmero, que atingiram 91,3\% de bons e excelentes de acordo com os critérios da UCLA $^{(25)}$ e $97,8 \%$ de consolidação ${ }^{(23)}$. Além disso, associamos amarrilhos com fios de sutura inabsorvível de poliéster trançado $n^{0} 5$ através de orifícios na parte proximal da placa, uma vez que, normalmente, o fragmento proximal, além da osteopenia, apresenta-se com reabsorção óssea e, com essa amarria, melhora-se sua fixação. Bosworth $^{(21)}$, foi quem inicialmente descreveu o tratamento desse tipo de pseudartrose com OI com placa angulada. Esta requer menor dissecção de tecidos moles, diminuindo inclusive o risco de necrose da cabeça do úmero $^{(22)}$. Em 1994, Jupiter e Mullaji ${ }^{(11)}$ relataram 88\% de consolidação em nove casos, porém, obtiveram $44 \%$ de resultados insatisfatórios com o uso de placa angulada e enxerto ósseo esponjoso. No entanto, salientaram ainda outras vantagens com relação à placa, como a resistência imposta pela lâmina à tração pela gravidade no foco de fratura e a possibilidade de compressão axial e interfragmentária exercida pelos parafusos distais. Em 2001, Ring et $\mathrm{al}^{(16)}$, utilizaram a placa angulada associada a enxerto esponjoso para OI dos casos de pseudartrose e obtiveram $80 \%$ de bons e excelentes resultados, de acordo com a escala de Constant-Murley modificada, porém, não ocorreu a consolidação em dois casos (8\%). Os autores correlacionam tal fato à condição clínica debilitada desses pacientes. Galatz et $\mathrm{al}^{(18)}$, em 2004, em 13 casos operados, com fixação utilizando placa angulada (10 casos) ou em "T" (três casos), obtiveram 12 resultados excelentes e satisfatórios, de acordo com os critérios de Neer ${ }^{(19)}$.

Em nossa casuística, obtivemos $61 \%$ de resultados excelentes e bons, de acordo com a escala funcional da UCLA, e $92 \%$ de consolidação. Não encontramos resultado ruim em nenhum dos casos e os regulares se devem a dor ocasional, limitação da amplitude de movimento e força motora grau $4 \mathrm{em}$ dois casos $(2,7)$. O caso 10 , tratava-se de um paciente que sofreu uma fratura do tubérculo maior e do acrômio, evoluindo com pseudartrose 
dos dois. Foi operado do acrômio e, quanto ao tubérculo maior, não foi possível sua reparação por completo. Após seis meses, teve um novo trauma, sofrendo uma fratura do colo cirúrgico do úmero, que também evoluiu para pseudartrose, sendo tratada com a técnica proposta. O caso 11 evoluiu com impacto subacromial ocasionado pela placa; tinha dor em atividades específicas e elevação do membro de $120^{\circ}$, porém, o paciente optou por não retirá-la. Por último, o caso 13, no qual se suspeitou de não consolidação da pseudartrose, sendo reoperado e colocado enxerto ósseo esponjoso na falha medial encontrada; tinha dor ocasional e $120^{\circ}$ de elevação. Apesar desses cinco casos regulares, todos os pacientes encontraram-se satisfeitos com o procedimento cirúrgico. Na literatura, encontramos somente dois casos de necrose avascular da cabeça do úmero após o tratamento da pseudartrose do colo do úmero com OI. Em nossa casuística, não verificamos nenhum caso de necrose da cabeça do úmero no período pós-operatório. Entretanto, como avaliamos somente os casos decorrentes de fraturas em duas partes do colo cirúrgico e em três partes, com desvio menor que cinco milímetros do tubérculo maior, de acordo com os critérios de $\mathrm{Neer}^{(24)}$, esperava-se que não houvesse desenvolvimento de necrose nesses casos (Tabela 1).
Tivemos ainda, como complicação, um caso de soltura parcial da placa em sua fixação distal, diagnosticada no terceiro mês de pós-operatório, mas a pseudartrose estava consolidada e a paciente encontrava-se sem queixa clínica (UCLA = 29) (caso 1). Em nossa opinião, a soltura ocorreu devido à osteopenia da diáfise e, para evitarmos novas complicações como essa, utilizamos a porca $P F O^{\circledR *}$, necessária em cinco de nossos casos (Tabela 1).

\section{CONCLUSÕES}

O tratamento cirúrgico da pseudartrose do colo cirúrgico do úmero pela técnica de redução aberta e OI com a placa angulada $P F S 80^{\circledR(23)}$, associada a enxerto ósseo tricortical intramedular e enxerto ósseo esponjoso autólogo, proporcionou a consolidação de $92 \%$ dos casos, além de resultados excelentes e bons em $61,5 \%$ dos pacientes e satisfação por parte de todos ao final do tratamento.

As pseudartroses do colo cirúrgico do úmero decorrentes de fraturas em duas partes tiveram melhor evolução, com $70 \%$ de resultados excelentes e bons, do que as decorrentes de fraturas em três partes envolvendo o colo cirúrgico e o tubérculo maior, que atingiram somente $33 \%$.

\footnotetext{
* Checchia SL, Mercadante MT, Santos PD, Miyazaki NA, Roncetti R, Klausser RG, Waldrigues MG. Desenvolvimento e avaliação preliminar de uma porca para fixação óssea. Tema livre apresentado no $32^{\circ}$ Congresso da Sociedade Brasileira de Ortopedia e Traumatologia realizado de 1 a 4 de novembro de 2000 no Rio de Janeiro.
}

\section{REFERÊNCIAS}

1. Checchia SL, Doneux PS, Miyazaki AN, Spir IA, Bringel R, Ramos CH. Classification of non-unions of the proximal humerus. Int Orthop. 2000;24(4):217-20.

2. Volgas DA, Stannard JP, Alonso JE. Nonunions of the humerus. Clin Orthop Relat Res. 2004;(419):46-50.

3. Lin J, Hou SM. Locked-nail treatment of humeral surgical neck nonunion. J Trauma. 2003;54(3):530-5.

4. Nayak NK, Schickendantz MS, Regan WD, Hawkins RJ. Operative treatment of nonunion of surgical neck fractures of the humerus. Clin Orthop Relat Res. 1995;(313):200-5.

5. Duralde XA, Flatow EL, Pollock RG, Nicholson GP, Self EB, Bigliani LU. Operative treatment of nonunions of the surgical neck of the humerus. J Shoulder Elbow Surg. 1996;5(3):169-80.

6. Wang GJ, Reger SI, Stamp WG. Nonunion of fractures of the proximal humerus: a method of treatment using a modified Moe plate. South Med J. 1977;70(7):818-20.

7. Sorensen KH. Pseudarthosis of the surgical neck of the humerus: two cases, one bilateral. Acta Orthop Scand. 1964;34:132-8.

8. Walch G, Badet R, Nové-Josserand L, Levigne C. Nonunions of the surgical neck of the humerus: surgical treatment with an intramedullary bone peg, internal fixation, and cancellous bone grafting. J Shoulder Elbow Surg. 1996;5(3):161-8.

9. Scheck M. Surgical treatment of nonunions of the surgical neck of the humerus. Clin Orthop Relat Res 1982;(167):255-9.

10. Antuña AS, Sperling JW, Sánchez-Sotelo J, Cofield RH. Shoulder arthroplasty for proximal humeral nonunions. J Shoulder Elbow Surg. 2002;11(6):114-21.

11. Jupiter JB, Mullaji AB. Blade plate fixation of proximal humeral non-unions. Injury. 1994;25(5):301-3.

12. Baux S, Razemon JP. Les fractures et les fractures-luxations de l'extrêmité supérieure de l'humerus. Rev Chir Orthop Reparatrice Appar Mot. 1969;55(5):387-496.

13. Healy WL, Jupiter JB, Kristiansen TK, White RR. Nonunion of the proximal humerus. A review of 25 cases. J Orthop Trauma. 1990;4(4):424-31.
14. Cofield RH. Comminuted fractures of the proximal humerus. Clin Orthop Relat Res. 1988;(230):49-57.

15. Coventry MB, Laumen EL. Ununited fractures of the middle and upper humerus. Special problems in treatment. Clin Orthop Relat Res. 1970;(69):174-8.

16. Ring D, McKee MD, Perey BH, Jupiter JB. The use of blade plate and autogenous cancellous bone graft in the treatment of ununited fractures of the proximal humerus. J Shoulder Elbow Surg. 2001;10(6):501-7.

17. Rockwood CA, Pearce JC. Management of proximal humerus nonunion. Orthop Trans. 1989;13:644.

18. Galatz LM, Willians GR Jr, Fenlin JM Jr, Ramsey ML, lannotti JP. Outcome of open reduction and internal fixation of surgical neck nonunions of the humerus. J Orthop Trauma. 2004;18(2):63-7.

19. Neer CS 2nd. Nonunion of the surgical neck of the humerus. Orthop Trans. 1983;7:389.

20. Norris TR, Turner JA, Bovil D. Nonunion of the upper humerus: an analysis of the etiology and treatment in 28 cases. In Post M. Morrey BF \& Hawkins RJ (ed): Surgery of the shoulder, Chicago, Mosby 1990 p. 63-7.

21. Bosworth DM. Blade plate fixation: technique suitable for fractures of the surgical neck of humerus and similar lesions. J Am Med Assoc. 1949;141(16):1111-3.

22. Galatz LM, lannotti JP. Management of the surgical neck nonunions. Orthop Clin of North Am. 2000;31(1):51-61.

23. Checchia SL, Doneux PS, Miyazaki AN, Fregoneze M, Silva LA, Lobo AC, et al. Avaliação do tratamento cirúrgico da fratura em duas partes do colo cirúrgico do úmero com placa PFS $80^{\circledR}$. Rev Bras Ortop. 2004;39:555-67.

24. Neer CS 2nd. Displaced proximal humeral fractures. Part I-Classification. J Bone Joint Surg Am. 1970;52(6):1077-89.

25. Ellman H, Kay SP. Arthroscopic subacromial decompression for chronic impingement. Two-to five-years results. J Bone Joint Surg Br. 1991;73(3):395-8.

26. Hawkins RJ, Bokor DJ. Clinical evaluation of shoulder problems. In: Rockwood CA Jr, Matsen FA 3rd. The Shoulder. Philadelphia: WB Saunders; 1998. p.175-180. 